\title{
QUISTE ÓSEO SIMPLE EN EL CALCÁNEO: REPORTE DE UN CASO.
}

\author{
Cevallos Peñaherrera Fabián Guillermo ${ }^{1}$, Llerena Freire Luis Francisco ${ }^{2 *}$, Benavides Vargas Karen Estefanía ${ }^{3}$, \\ Álvarez Guerrero Daniel Alejandro ${ }^{3}$, Heredia Montenegro Cristina Elizabeth ${ }^{3}$, Freire Pazmiño Dennise Solange ${ }^{3}$, \\ Llerena Freire Giannella Izamar'. \\ DOI: $10.48018 / \mathrm{rmv} . v 31 . i 2.14$
}

1. Hospital General IESS Ambato. Médico Tratante del Servicio de Traumatología y Ortopedia. Ambato Ecuador.

2. Hospital General IESS Ambato. Médico Residente

del Servicio de Traumatología y Ortopedia. Ambato

Ecuador

3. Universidad Técnica de Ambato. Facultad de

Ciencias de la Salud. Carrera de Medicina. Ambato -

Ecuador.

4. Universidad de las Américas. Facultad de Medicina. Carrera de Medicina. Quito - Ecuador.

ORCID ID:

Cevallos Peñaherrera Fabián Guillermo: orcid.org/0000-0002-8601-0626

Llerena Freire Luis Francisco

orcid.org/0000-0002-8383-4099

Benavides Vargas Karen Estefanía:

orcid.org/0000-0001-5543-6686

Álvarez Guerrero Daniel Alejandro: orcid.org/0000-0003-0301-099

Heredia Montenegro Cristina Elizabeth:

orcid.org/0000-0003-0963-6294

Freire Pazmiño Dennise Solange:

orcid.org/0000-0002-2807-2183

Llerena Freire Giannella Izamar:

orcid.org/0000-0001-9404-2030.

*Corresponding author: Llerena Freire Luis

Francisco

E-mail: pancho_llerena@hotmail.com

Article history

Received: 07 - Ago - 2020

Accepted: 20 - Oct - 2020

Publish: 01 - Nov - 2020

CARE 2017 Check List statement: The authors have real the CARE 2017 Check List and the

manuscript was prepared and revised according to the CARE 2017 Checklist.

Conflict of interest: All authors declared that there are no conflicts of interest.

Financial disclosure: The authors have no financial relationships relevant to this article to disclose.

Authors' contribution: All the authors contributed in the search, selection of articles and writing. All the authors reviewed and approved the final manuscript.

Forma de citar este artículo: Cevallos Peñaherrera FG, Llerena Freire LF, Benavides Vargas KE, Álvarez Guerrero DA, Heredia Montenegro CE, Freire Pazmiño DS, et al. QUISTE ÓSEO SIMPLE EN EL CALCÁNEO: REPORTE DE UN CASO. Rev Med Vozandes. 2020; 31 (2): $101-106$

\section{Resumen}

Las tumoraciones óseas son patologías producto de modificaciones en la estructura del hueso y en su contenido, pueden presentarse como lesiones tumorales benignas o malignas, primitivas o metastásicas, en dependencia de varios factores: la edad de presentación, localización, velocidad de crecimiento, reacción perióstica e infiltración a estructuras vecinas. Las publicaciones demuestran que los quistes óseos simples asintomáticos y pequeños no requieren tratamiento, hasta el $25 \%$ son de resolución espontánea posterior a una fractura patológica; mientras que quistes más grandes con corteza delgada, requieren de algún tipo de intervención.

Se presenta el caso de una paciente de sexo femenino de 17 años de edad, que acude por presentar dolor de moderada intensidad en talón izquierdo que se intensifica a la deambulación, con un tiempo de 2 meses de evolución, posterior a un trauma contuso en esta región. Al examen físico se evidencia dolor a la digito presión y maniobras de movilización activa-pasiva en cara lateral externa a nivel de calcáneo de pie izquierdo. Se realiza radiografía lateral simple de calcáneo izquierdo, donde se evidencia una lesión lítica circular de 3 por $3 \mathrm{~cm}$ que compromete la región anteroinferior de calcáneo.

En conclusión, el quiste óseo simple o solitario, es una patología benigna, que, aunque no ponga en peligro la vida de la persona puede afectar seriamente la funcionalidad del pie. En la actualidad no existe un tratamiento estandarizado para el manejo de esta patología, las técnicas quirúrgicas actuales en especial el curetaje y el autoinjerto óseo resultan muy prometedoras.

Palabras clave: Apendicitis del muñón, apendicectomía laparoscópica, muñón apendicular, reporte de caso.

\section{Abstract \\ SIMPLE OSSEOUS CYST OF THE CALCANEUS A CASE REPORT.}

Bone tumors are pathologies resulting from modifications in the bone structure and its content, they can present as benign or malignant, primitive or metastatic tumor lesions, depending on various factors: age of presentation, location, growth rate, periosteal reaction and infiltration to neighboring structures. The publications show that small, asymptomatic and small bone cysts do not require treatment, up to $25 \%$ are spontaneous resolution after a pathological fracture; while larger cysts with thin bark require some type of intervention.

The case of a 17-year-old female patient is presented, who presents for presenting moderate intensity pain in the left heel that intensifies on ambulation, with a time of 2 months of evolution, after a blunt trauma in this region. On physical examination, pain from the pressure digit and active-passive mobilization maneuvers were evidenced on the external lateral aspect at the calcaneus level of the left foot. A simple 


\section{Abstract}

lateral radiograph of the left calcaneus was performed, showing a 3 by $3 \mathrm{~cm}$ circular lytic lesion involving the anteroinferior region of the calcaneus.

In conclusion, simple or solitary bone cyst is a benign pathology that, although it does not endanger the person's life, can seriously affect the functionality of the foot. Currently, there is no standardized treatment for the management of this pathology, current surgical techniques, especially curettage and bone autograft are very promising.

Keywords: Bone cyst, benign pathology, calcaneus, diagnosis and treatment

\section{Introducción}

Las lesiones óseas tumorales constituyen patologías que son producto de modificaciones en la estructura del hueso y en el contenido del mismo, pueden presentarse como lesiones tumorales benignas o malignas, primitivas o metastásicas, en dependencia de varios factores; tales como, la edad de presentación, localización, velocidad de crecimiento, reacción perióstica e infiltración a estructuras vecinas. ${ }^{1}$ Estos tumores se localizan en diferentes regiones del esqueleto, representando del 1 al 5\% del total los localizados en el pie y el tobillo; de los cuales, el $2 \%$ aparecen en el pie y son benignos en la mayoría de casos. De los tumores localizados en el pie, la región de predominio es la parte media y posterior del pie. ${ }^{2}$

Dentro de los tumores óseos benignos, tenemos al quiste óseo simple, también conocido como solitario, que representa el $3 \%$ de los tumores óseos primarios, definido por la OMS como una cavidad unicameral llena de líquido transparente o serosanguíneo, cubierta por una membrana de tejido conectivo vascular, en la que se visualizan células osteoclásticas dispersas y zonas hemorrágicas o cristales de colesterol, tiene predominio por la metástasis de los huesos largos principalmente el húmero y fémur y es rara su localización en el calcáneo. ${ }^{3}$ El quiste óseo simple del calcáneo representa el 2 al 14\% de los quistes óseos simples, aparece entre la segunda y tercera década de la vida, con una relación hombre y mujer de 2:1.4

Es una patología rara y la mayoría de los casos suele presentarse de forma asintomática; por lo que, puede pasarse por alto, algunos pacientes experimentan un dolor localizado cuya intensidad va progresando acompañado en ocasiones de edema, este dolor se da por fracturas corticales y trabeculares, que se producen cuando el tamaño del quiste sobrepasa el $1 / 3$ de su masa. El diagnóstico es incidental o se da posterior a la presencia de una fractura patológica, en la radiografía se visualiza como una lesión lítica localizada que presenta márgenes escleróticos delgados, bien definidos en el canal medular sin reacción perióstica, que puede expandirse concéntricamente. ${ }^{5}$

Los estudios manifiestan que los quistes óseos simples asintomáticos y pequeños no requieren tratamiento; el $25 \%$ se resuelven espontáneamente durante la maduración esquelética o posterior a una fractura patológica; Mientras que los más grandes de corteza delgada necesitan ser intervenidos. ${ }^{6}$ Para lo cual existen diversos tratamientos que pueden ser aplicados: procedimientos de mínima invasión como la infiltración con corticoesteroides y otros de mayor complejidad principalmente la realización de un legrado más colocación de injerto óseo ya sea autólogo, heterólogo o sintético (polimetacrilato o hidroxiapatita). Otra opción terapéutica es la inyección percutánea intralesional con médula ósea autóloga. ${ }^{7}$

El objetivo de este trabajo es presentar un caso clínico de quiste óseo simple localizado en el hueso calcáneo, en el cual se discuten los aspectos característicos de la patología y se realiza una breve revisión sobre el tratamiento más adecuado.

El estudio sigue las recomendaciones CARE, para el reporte de casos clínicos, además, el paciente firmo el consentimiento informado autorizando su publicación.

\section{Presentación del caso}

Paciente de sexo Femenino de 17 años, sin antecedentes clínicos, quirúrgicos ni familiares de importancia. Acude a consulta externa del servicio de Traumatología por cuadro de 2 meses de evolución caracterizado por presentar dolor de moderada intensidad en talón izquierdo que se intensifica a la deambulación, como causa aparente trauma contuso en esta región. Al examen físico en pie izquierdo: talón, se evidenció dolor a la digito presión y a las maniobras de movilización activa-pasiva en cara lateral externa a nivel del calcáneo. Se realizó una radiografía lateral simple del calcáneo izquierdo con sospecha clínica de espolón, donde se evidenció una lesión lítica circular de 3 por $3 \mathrm{~cm}$ que compromete la región anteroinferior del calcáneo. (Figura 1A)

Dado el hallazgo fue ingresada a hospitalización para estudio del caso. Los exámenes de laboratorio estuvieron dentro de los parámetros normales. Se realizó una resonancia magnética en la secuencia coronal y sagital, donde se observó un quiste de paredes delgadas con líquido en su interior, localizado en la región anteroinferior del calcáneo. (Figura 2A y 2B) Se 


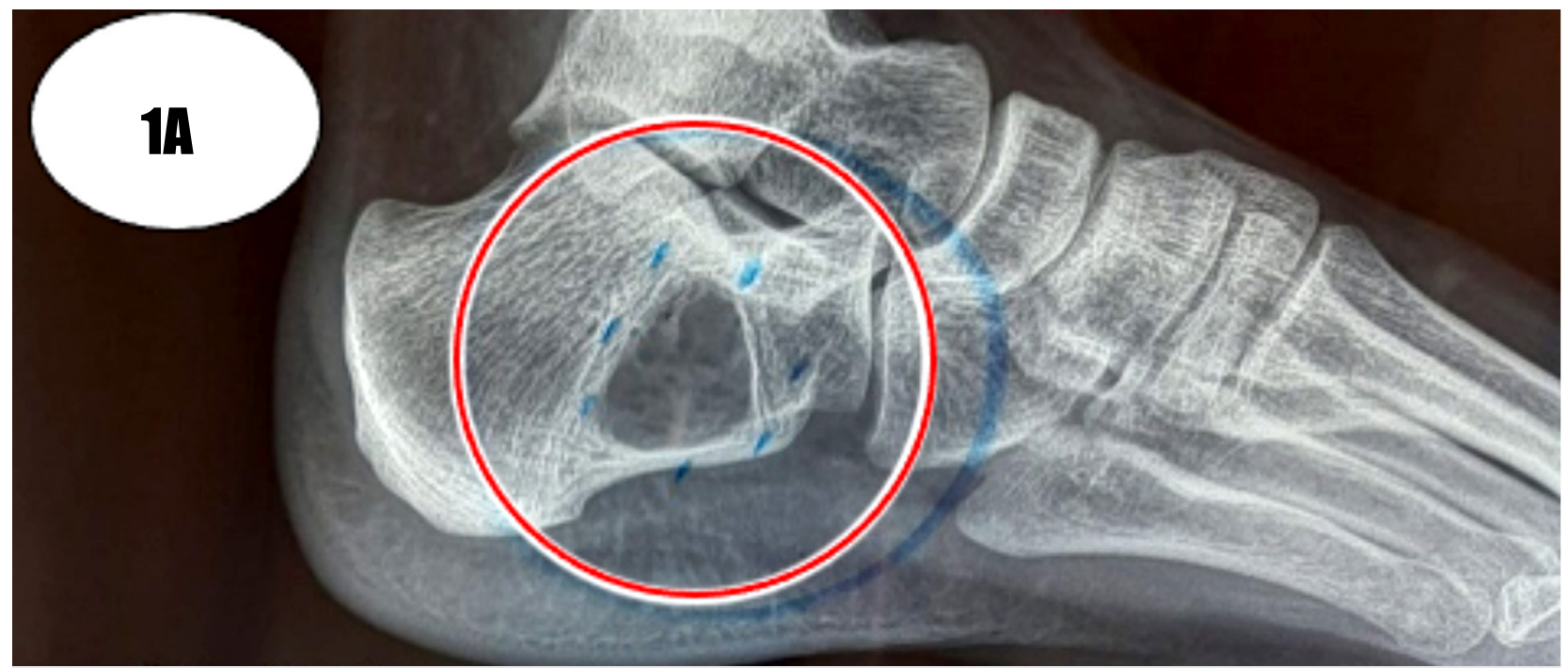

Figura 1 . Radiografía simple de calcáneo izquierdo.
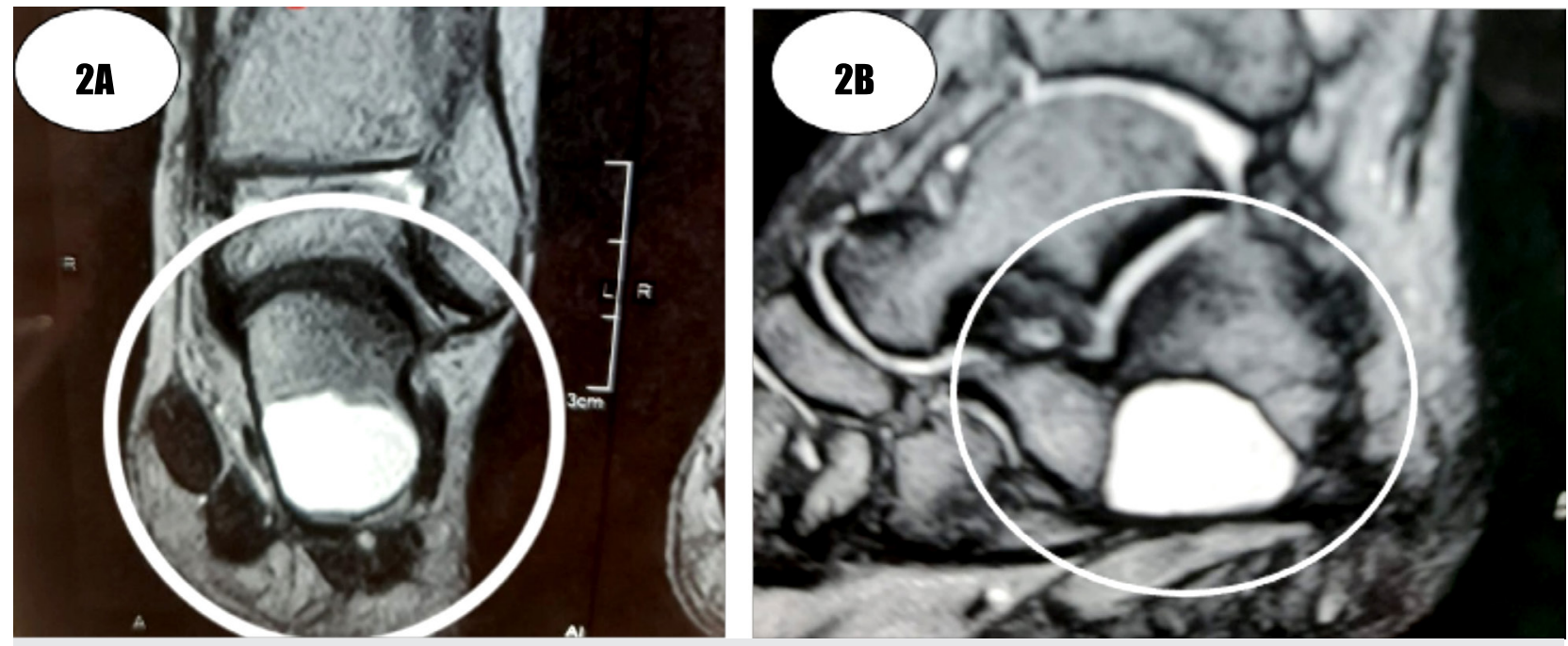

Figura 2A, 2B . Resonancia magnética del calcáneo

planteó como diagnóstico inicial un quiste óseo simple en el calcáneo, por el tamaño y el riesgo de colapso se decidió resolución quirúrgica.

El procedimiento quirúrgico se llevó a cabo con el paciente en decúbito dorsal y con la utilización de un torniquete hemostático a nivel de región proximal de la pantorrilla izquierda, se efectuó una incisión en cara lateral de calcáneo de $5 \mathrm{~cm}$ de longitud que compromete piel y tejido celular subcutáneo. (Figura 3A) Luego se realizó una ventana ósea en la cortical externa del calcáneo de $1 \times 1 \mathrm{~cm}$ pudiendo visualizarse el quiste óseo, se drenó obteniendo $5 \mathrm{ml}$ de líquido amarillento, posteriormente se realizó curetaje óseo de la cavidad quística y lavado de la misma con abundante suero fisiológico. A continuación, se efectuó una incisión en cara anterior de cresta iliaca izquierda de $4 \mathrm{~cm}$ de longitud que compromete piel, tejido celular subcutáneo y músculo; se obtuvo injerto óseo esponjoso (autólogo); el mismo que, se colocó en cavidad quística de calcáneo. Finalmente se hizo el cierre de heridas quirúrgicas por planos.

El reporte histopatológico confirmó que se trataba de un quiste óseo simple. El manejo posquirúrgico en esta paciente fue antibioticoterapia profiláctica quirúrgica (Cefazolina l gramo vía intravenosa cada 8 horas por 3 días), analgesia (Paracetamol 1 gramo vía intravenosa cada 8 horas por 3 días, Ketorolaco 30 miligramos vía intravenosa cada 8 horas por 2 días) e hidratación (Lactato Ringer 1000 mililitros vía intravenosa $60 \mathrm{ml} / \mathrm{h}$ por 3 días). Se utilizó el método RICE (reposo, hielo, compresión y elevación) de miembro inferior izquierdo. Se realizó control radiográfico posquirúrgico. (Figura 4A y 4B)

Al tercer día sin evidencia de complicación ulteriores de sitio quirúrgico paciente fue dada de alta. Se indicó uso de muletas por 4 semanas, 
posterior a ese tiempo se permitió apoyo parcial e inicio de fisioterapia. Al tercer mes posquirúrgico, paciente con apoyo total de miembro inferior izquierdo, movilidad conservada, se realizó control radiográfico donde se evidencia aumento de trabeculación en zona quística. (Figura 5A y 5B)

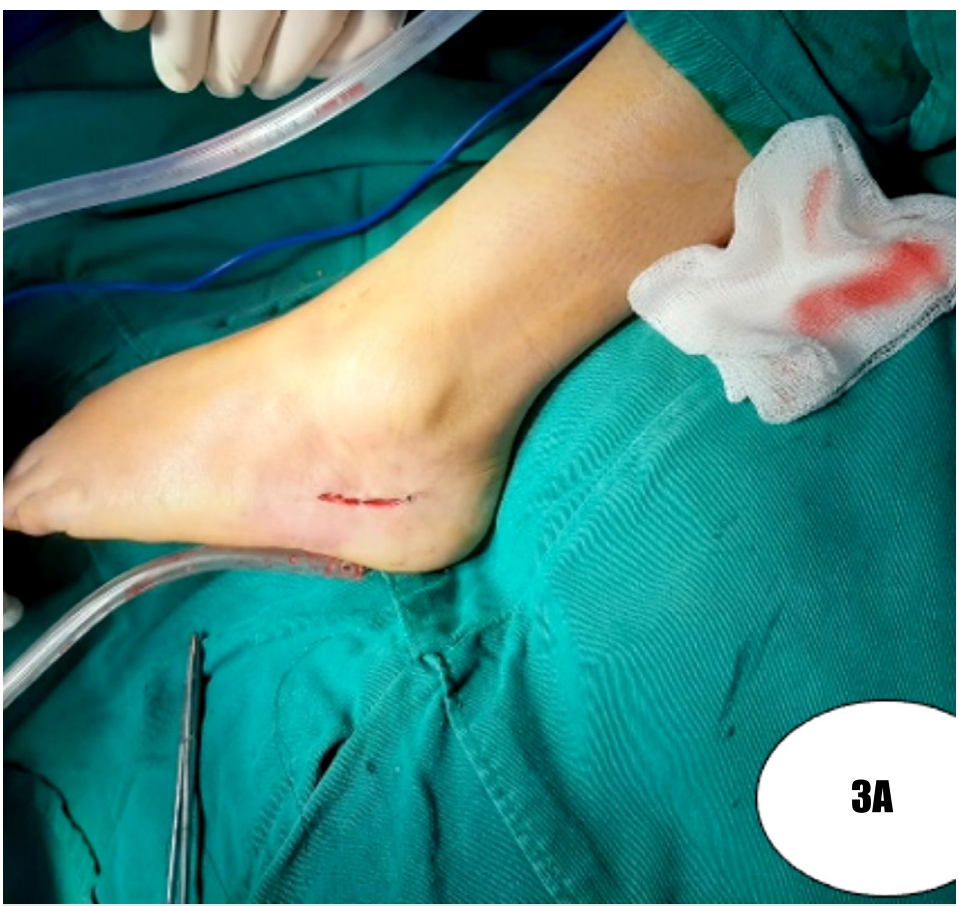

Figura 3A . Incisión lateral externa en región de calcáneo

Fuente: Elaborado por los autores

\section{Discusión}

Los huesos son estructuras anatómicas constituidas por varios minerales como el calcio, fosforo y potasio, forman parte del sistema osteomioarticular en conjunto con cartílagos, músculos y ligamentos. Los tumores que se evidencian en un hueso, pueden ser verdaderamente óseos, cartilaginosos o por resorción, el hueso puede cambiar su mineralización y secundario a esto presentar modificaciones en su densidad.
Se define como quiste óseo simple al tumor benigno que no presenta tejido epitelial de revestimiento, la Organización Mundial de la Salud lo clasifica como lesiones de células gigantes y quistes óseos que aparecen en la edad pediátrica, que se caracteriza por ser únicompartimental en su gran mayoría. La incidencia de esta patología es mayor en pacientes masculinos en un rango de edad entre los 10 y 25 años.?

El quiste óseo solitario se localiza frecuentemente en la metáfisis de los huesos largos, la evidencia reporta el mayor número de casos aproximadamente el $50 \%$ en el humero proximal, el $20 \%$ en el fémur proximal y muy infrecuentemente se encuentra en el calcáneo específicamente en el cuello del mismo. ${ }^{10,11} \mathrm{El}$ caso presentado en este artículo coincide con la revisión de la literatura ya que el hallazgo de esta condición patológica es incidental y aunque no pone en riesgo la vida del paciente, al verse afectado considerablemente el hueso calcáneo este puede perder la funcionalidad del miembro inferior ya que causa deformidad e incapacidad.

La etiología de esta patología es multifactorial, por causas traumáticas, inflamatorias y obstrucción de la irrigación venosa de los huesos. ${ }^{12}$ Otra de las causas que se conoce es una degeneración quística de algún tumor previo o una falla en el proceso normal de osificación. Las pruebas de imagen juegan un papel muy importante en el diagnóstico de esta enfermedad ya que como se conoce según la bibliografía la detección de la misma es incidental, en la radiografía que es el primer examen que se practica se puede observar lesiones líticas que se localizan a nivel central, en donde se puede o no diferenciar márgenes escleróticos, cuando esta condición se acompaña de una fractura patológica se puede apreciar un segmento óseo en la parte declive de la lesión, lo que se denomina como
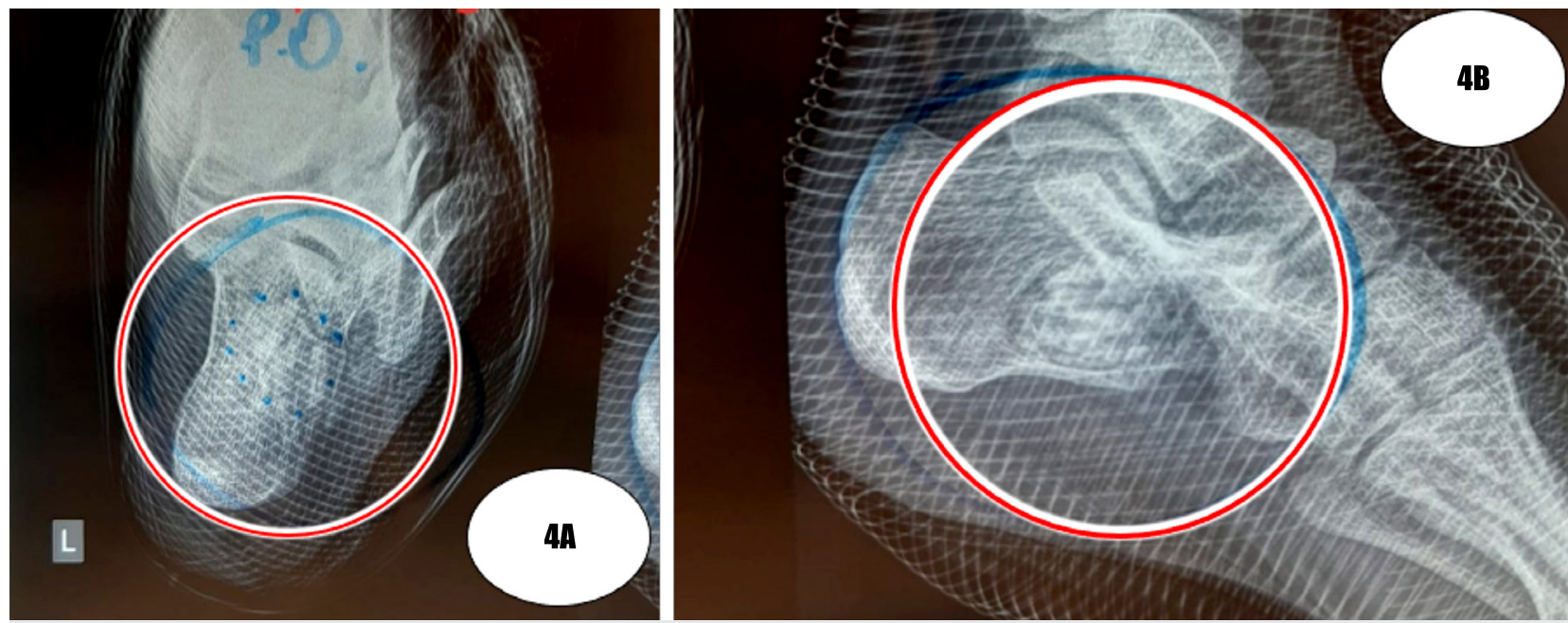

Figura 4A, 4B . Radiografía simple de calcáneo izquierdo. Primer día posquirúrgico 


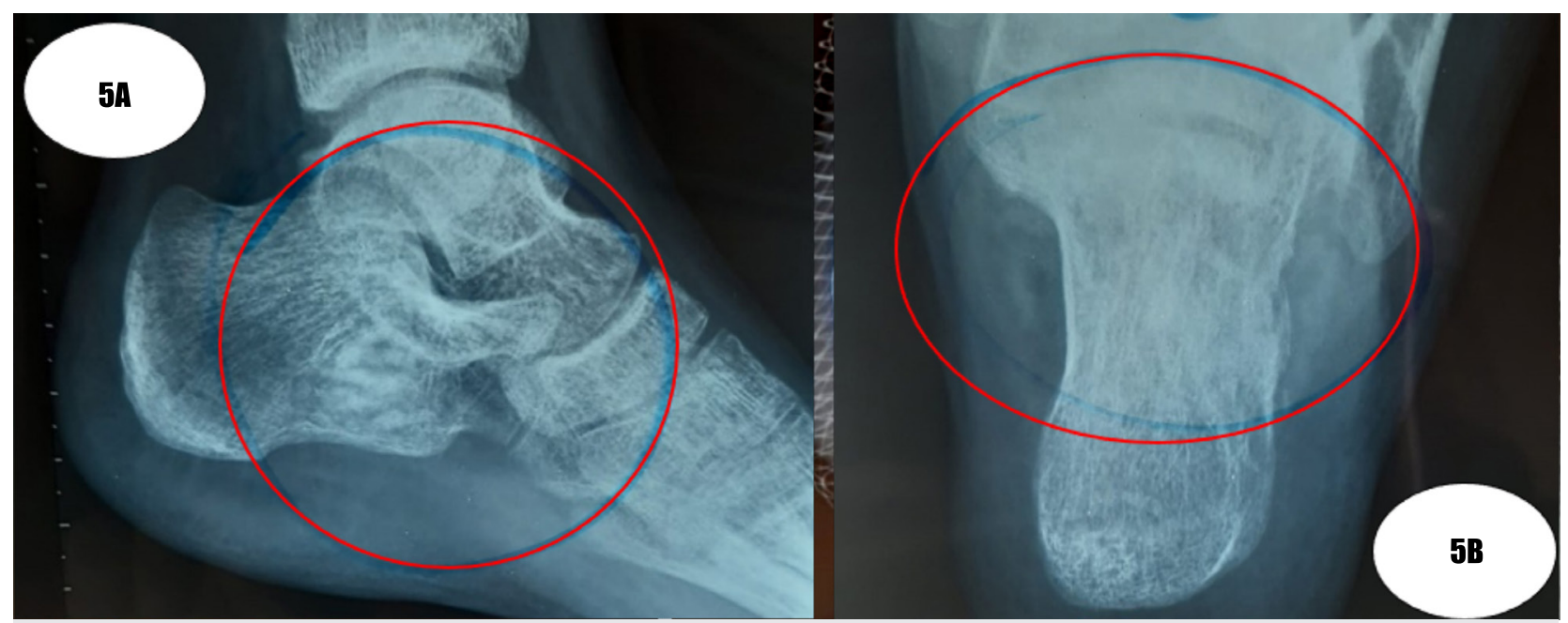

Figura 5A, 5B . Radiografía simple de calcáneo izquierdo. Tercer mes posquirúrgico

Fuente: Elaborado por los autores

el signo del segmento caído, aunque este hallazgo no es muy común se considera que es característico del quiste óseo simple.

La tomografía es otro estudio de imagen que se puede realizar ya que nos permite confirmar el diagnóstico, aunque este no se practica muy frecuentemente. La resonancia magnética nos permite identificar el origen quístico de la lesión sobre todo en los casos que se presentan de forma atípica, si la lesión está acompañada de una fractura puede evidenciarse niveles liquido-líquido y septos de tal manera que puede aparentar un quiste aneurismático, cuando en esta prueba diagnóstica observemos que el diámetro transversal de la lesión del hueso afectado es mucho más pequeño que el diámetro más ancho debemos considerar que se trata de un quiste óseo complicado. ${ }^{10}$

Los quistes óseos simples que han sufrido una fractura estable pueden evolucionar favorablemente con el uso de un inmovilizador, si la fractura es inestable o se encuentra cercana a una zona de carga es necesario colocar material de osteosíntesis para fijar la fractura. En el caso de que el quiste óseo simple haya sido un hallazgo incidental, se recomienda realizar un tratamiento quirúrgico con legrado e injerto óseo. ${ }^{10}$

El manejo del quiste óseo por medio del curetaje y colocación de injerto ha indicado dar buenos resultados a corto y largo plazo. Actualmente una alternativa al legrado abierto es la realización de un curetaje endoscópico, guiado por intensificador, ya que es un procedimiento poco invasivo y que proporciona a los pacientes un índice menor de complicaciones, una recuperación más rápida, una inserción temprana a la vida cotidiana y mejoría en la funcionalidad. ${ }^{6}$

Es recomendable realizar un lado de la cavidad quística con una solución de etanol al 95\% durante 2-3 minutos, previo a realizar el injerto garantizando que las membranas restantes del quiste se desnaturalicen. Luego de esto se debe realizar un lavado con solución salina y evitar que los tejidos blandos no entren en contacto con el etanol. En cuanto al injerto es recomendable usar hueso esponjoso para el plombage de la cavidad, ya que permite una aplicación fácil de las astillas alógenas del hueso esponjoso. ${ }^{13}$
Existe una recurrencia del $18,9 \%$ del quiste unicameral, en pacientes que han sido tratados con legrado endoscópico, en un periodo de entre 7,8 a 28,8 meses, dicha recurrencia fue asociada a la continuidad que exista entre el quiste y la fisis ósea $(p=0.006)$, del total de los pacientes se categorizaron como curados al 83,7\%; sin embargo la presencia de un residuo quístico fue significativa en pacientes menores de 10 años con una $p=0,034$, el residuo no fue encontrado en pacientes con quistes en el calcáneo. ${ }^{12}$

Hoy en día se encuentran disponibles otras alternativas de tratamiento que se han ido desarrollando con el tiempo, como la utilización de hidroxiapatita para llenar la cavidad que queda tras el drenaje del quiste solitario, permitiendo una osteo-integración total del material con los tejidos del paciente, brindando así una alternativa viable a los profesionales de la salud para el manejo de los pacientes con estas afecciones del sistema osteomioarticular. ${ }^{14}$ La hidroxiapatita es una alternativa a la utilización de cemento de fosfato de calcio, ambos componentes muestran una gran funcionalidad en periodos de 1 a 5 años con porcentajes altos de curación; sin embargo, la tasa de recurrencia fue sustancialmente más alta en niños menores de 10 años $(p=0,01)$ en quiste activos $(p=0,03) \quad(p<0,001)$ y ubicados en huesos largos $(p=0,01)(p<0,001) \cdot{ }^{15,11}$

Una nueva estrategia de tratamiento se basa en la utilización hueso diseñado con tejidos como una alternativa al manejo del quiste óseo con hueso alogénico; no obstante, no se han encontrado diferencias significativas entre la aparición de complicaciones y curación ósea $(p<0.05) .{ }^{16}$

En conclusión, el quiste óseo simple o solitario, es una patología ósea benigna, que, aunque no ponga en peligro la vida de la persona que lo padece puede afectar seriamente la funcionalidad del pie, el diagnóstico suele ser un hallazgo casual, en la mayoría de los casos tiene una evolución favorable posterior al tratamiento; aunque en la actualidad no existe un tratamiento estandarizado para el manejo 
de esta patología, las técnicas quirúrgicas actuales en especial el curetaje y el autoinjerto óseo resultan muy prometedoras. Lo mismo que puede ser evidenciado en el caso clínico presentado en este estudio.

\section{Referencias}

1. Maso M, Borrero C, Leguén M, Olivera R. Quiste óseo solitario. Presentación de un caso. Bayamo 2017. Multimed. 2019; 23(4): p. 812-822.

2. Gonzales C, Bolsa P, Mosquera A, Balboa V, Gulias D. Simple bones cyst in the calcaneus. About a case. Clin Case Studie Rep. 2019 Diciembre $31 ; 2$ : p. 1-4.

3. Chinze C, Kanzaki N, Fujishiro T, Hayashi S Hashimoto S, Kuroda R, et al. Arthroscopic debridement of a talar cyst and bone grafting with the osteochondral autograft transfer system. Journal of the American Podiatric Medical Association. 2017; 107(6): p. 541-547.

4. Malghem J, Lecouvet F, Vande B. Calcaneal cysts and lipomas: a common pathogenesis? Skeletal radiol. 2017;: p. 1-8.

5. Ahmed Z, Bin S, Ahmad N, Raza A. Hidden unicameral bone cyst of the calcaneus: a case report of military soldier with chronic ankle pain. J Arch Mil Med. 2017 Junio; 5(2): p. 1-3.

6. Zaghloul A, Haddad B, Khan W, Grimes L, Tucker K. A novel minimally invasive technique for treatment of unicameral bone cysts. The Open Orthopaedics Journal.
2015; 9: p. 475-479.

7. Fauzi A, Aprizal R, Prabowo Y. Simple bone cyst management: percutaneous steroid injection versus curettage with hydroxiapatite. Indonesian Journal of Cancer. 2017; 11 (2): p. 71-76.

8. Riley DS, Barber MS, Kienle GS, Aronson JK, von Schoen-Angerer T, Tugwell P, et al. CARE guidelines for case reports: explanation and elaboration document. j Clin Epidemiol. 2017. p: S0895-4356 (17) 30037-9.

9. Cruz R, Calderón V, Trevejo A, Edilbrando N. Control imagenológico de un quiste óseo simple: reporte de un caso. Estomatol Herediana. 2018;: p. 195-200.

10. Lerma J, De la Pedraja I, Lancharro, Monte Llanos D. Enfoque y manejo radiológico de las lesiones óseas líticas pediátricas. Sociedad Española de Radiología Medica. 2018;: p. 5-6.

11. Bukva B, Vrgoc G, Abramovic D, Dusic S, Brkic l. Treatment of Unicameral Bone Cysts in Children: A Comparative Study. Original Scientific Paper. 2019;: p. 403-409

12. Aiba H, Kobayashi M, Waguri-Nagaya
Y, Goto H, Mizutani J, Yamada S, et al. Treatment of simple bone cysts using endoscopic curettage: a case series analysis. Aiba et al. Journal of Orthopaedic Surgery and Research. 2018; 13(168).

13. Toepfer A, Lenze U, Gerdesmeyer L, Pohlig F, Harrasser N. Endoscopic resection and allografting for benign osteolytic lesions of the calcaneus. Toepfer et al. SpringerPlus. 2016 ; $5(427)$.

14. Loforte A, Torre M, Grey J, Rodríguez E. Tratamiento quirúrgico del quiste óseo solitario con el empleo de la hidroxiapatita. CCM. 2015; 19(4).

15. Higuchi T, Yamamoto N, Shirai T, Hayashi K, Takeuchi A, Kimura H, et al. Treatment outcomes of the simple bone cyst A comparative study of 2 surgical techniques using artificial bone substitutes. Medicine. 2018 Abril; 97(18).

16. Hou T, Wu Z, Xing J, Wu X, Luo F, Xie Z, et al, Tissue-engineered bone treating simple bone cyst-a new strategy. Journal of Surgical Research. 2016; 200(2). 